\title{
Glycogen-rich clear cell carcinoma of the breast with solid papillary pattern: Two cases with heterogeneous clinicopathological features
}

\author{
TIBERIU-AUGUSTIN GEORGESCU ${ }^{1,2}$, OCTAVIAN MUNTEANU ${ }^{3,4}$, ANTONIA-CARMEN LISIEVICI $^{2}$, \\ TIBERIU TEBEICA ${ }^{5}$, DRAGOȘ CRET,OIU ${ }^{6,7}$, OANA TOADER $^{8,9}$, \\ NICOLAE SUCIU ${ }^{8,9}$ and ROXANA ELENA BOHÎLT,EA ${ }^{3,9}$
}

\footnotetext{
${ }^{1}$ Department of Pathology, 'Alessandrescu-Rusescu' National Institute for Mother and Child Health, 020395 Bucharest;

${ }^{2}$ Discipline of Pathology, 'Carol Davila' University of Medicine and Pharmacy, 050474 Bucharest;

${ }^{3}$ Department of Obstetrics and Gynecology, Emergency University Hospital of Bucharest, 050098 Bucharest;

${ }^{4}$ Discipline of Anatomy, 'Carol Davila' University of Medicine and Pharmacy, 050474 Bucharest;

${ }^{5}$ Department of Pathology, Dr Leventer Centre, 011216 Bucharest; ${ }^{6}$ Department of Genetics,

'Alessandrescu-Rusescu' National Institute for Mother and Child Health, 020395 Bucharest; ${ }^{7}$ Discipline of Histology,

'Carol Davila' University of Medicine and Pharmacy, 050474 Bucharest; ${ }^{8}$ Department of Obstetrics and Gynecology,

'Alessandrescu-Rusescu' National Institute for Mother and Child Health, 020395 Bucharest;

${ }^{9}$ Discipline of Obstetrics and Gynecology, 'Carol Davila' University of Medicine and Pharmacy, 050474 Bucharest, Romania
}

Received January 8, 2021; Accepted February 9, 2021

DOI: $10.3892 /$ etm.2021.9956

\begin{abstract}
Invasive breast cancer is a heterogeneous disease, both in its histopathological classification and clinical course. Glycogen-rich clear cell carcinoma of the breast is an extremely rare subtype of invasive breast carcinoma, accounting for up to $3 \%$ of all breast carcinomas. The tumor is composed of polygonal cells with abundant clear cytoplasm containing glycogen and has a very controversial prognosis. Solid papillary pattern is an uncommon morphological variant of breast carcinoma which is associated with indolent behavior in the absence of an invasive component. To date, there are only three cases of glycogen-rich clear cell carcinoma with solid papillary pattern reported in the English literature. In this article, we present two cases of glycogen-rich clear cell carcinoma of the breast, encountered in our daily clinical practice over a period of 5 years (2015-2020) and perform a brief review of currently published literature. Unlike most cases of glycogen-rich clear cell carcinoma documented to date, follow-up of our case featuring solid papillary pattern revealed extremely favorable
\end{abstract}

Correspondence to: Dr Octavian Munteanu, Discipline of Anatomy, 'Carol Davila' University of Medicine and Pharmacy, 8 Eroilor Sanitari Boulevard, 050474 Bucharest, Romania

E-mail: octav_munteanu@yahoo.com

Key words: glycogen-rich breast carcinoma, clear cell breast carcinoma, solid papillary carcinoma, invasive breast carcinoma, rare breast cancer subtype clinical outcome, suggesting a better prognosis for tumors with this morphology.

\section{Introduction}

Invasive breast carcinoma of no special type (IBC-NST) comprises a large and heterogenous group of invasive breast carcinomas lacking the morphological hallmarks of special histologic types (1). IBC-NST is the most common histological subtype, accounting for $80 \%$ of all invasive breast carcinomas (2). In recent years, several morphologic patterns of IBC-NST have been recognized and most of them have also been acknowledged in the latest classification of the World Health Organization (WHO). These include: Carcinomas with oncocytic, sebaceous, lipid-rich, glycogen-rich/clear cell, medullary, neuroendocrine, pleomorphic, osteoclast-like giant cells, choriocarcinomatous and melanocytic features/patterns (2).

Glycogen-rich/clear cell carcinoma (GRCCC) of the breast has some common features with clear cell carcinomas of other organs. It was first described by Hull et al in 1981 (3) and its incidence has been estimated as less than $3 \%$. According to the WHO classification of tumors, GRCCC of the breast is defined as a rare subtype of IBC-NST in which more than $90 \%$ of the tumor cells have abundant clear cytoplasm containing glycogen. To date, the clinicopathologic and biologic features of GRCCC are yet to be described and clinical outcome is still unclear. Some authors hypothesized that it may be similar to other breast carcinomas when compared on a stage-matched basis (4), while others indicated a less favorable outcome for patients with GRCCC $(5,6)$.

In this article, we report two cases of GRCCC of the breast encountered in our clinical practice over a period of 
5 years (2015-2020), featuring a similar immunophenotype, but completely different morphological patterns and patient outcome.

\section{Case report 1}

We encountered the first case of GRCCC in our daily clinical practice in September 2015, when a 64-year-old female patient with no family history of breast cancer, presented with a lump in the left breast. Initial physical examination revealed a palpable, tender and irregular mass with retraction of the overlying epidermis, located in the upper-outer quadrant of the left breast. Ultrasonography revealed an irregular, hypoechoic breast tumor with heterogeneous internal echoes, qualifying as BI-RADS 5. The core needle biopsy was signed out as infiltrative breast carcinoma with clear cells, defer to excision for final classification. The patient underwent total mastectomy with axillary lymph node dissection and the specimen was sent to our Department of Pathology for histopathological examination. A white-tan, spiculated tumor mass measuring 46/32/28 mm was identified in the upper-outer quadrant of the breast.

Histologically, the tumor was composed of polygonal epithelial cells with well-defined borders and strikingly clear or finely-granular cytoplasm, arranged in invasive nests, cords and/or trabecular structures, separated by thin fibrous septa (Fig. 1A). Tumor cell nuclei had irregular shapes and sizes, featuring granular chromatin pattern and prominent mitotic figures (Fig. 1B). The average mitotic index was 17 mitoses per $10 \mathrm{HPF}$.

Focal lympho-vascular infiltration was noted and macrometastases were identified in four of the 12 ipsilateral axillary lymph nodes examined. Periodic acid-Schiff staining method employing diastase confirmed the presence of cytoplasmic glycogen granules (Fig. 1C and D).

Immunostaining with myoepithelial markers revealed no intraductal component within or at the periphery of the tumor. Across the entire proliferation, the tumor cells were estrogen receptor (ER)-positive (Fig. 1E), progesterone (PR)-negative and HER2-negative (score 0). Ki67 proliferation index was $25 \%$. E-cadherin revealed crisp membranous positivity in all tumor cells and p53 was normal, showing 'wild-type' pattern of expression. The cytokeratin panel revealed positivity for CK7 and CK8/18.

Based on these findings, the morphological features and immunohistochemical phenotype supported the diagnosis of glycogen-rich clear cell carcinoma of the breast, pT1c pN2a.

A postsurgical full-body CT revealed sclerotic lesions located in the liver and third and fourth lumbar spine vertebrae (L3-L4). The patient underwent eight cycles of chemotherapy, but unfortunately succumbed to the disease after 7 months.

\section{Case report 2}

The second case we encountered was diagnosed in January 2020, in a 54-year-old woman who underwent simple subcutaneous mastectomy accompanied by sentinel lymph node excision for a clinically palpable left/right breast mass. The lesion was previously diagnosed as IBC-NST on core needle biopsy. A subcutaneous simple mastectomy specimen,

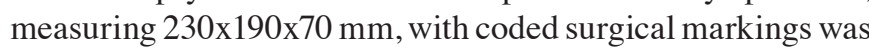
received in buffered formalin. The posterior resection margin was inked black and the specimen was serially sectioned at 5-mm intervals. Upon gross examination, the tumor mass was located in the outer-upper quadrant, measured 18/16/12 mm and appeared to be well-circumscribed, with heterogeneous appearance, featuring tan and hemorrhagic areas. The whole tumor was embedded, including surrounding fibrous areas and resection margins. Complete submission of the sentinel lymph node for routine histology and immunohistochemistry was performed, according to standard protocols. All sections were stained with hematoxylin and eosin (H\&E). Periodic acid Schiff stain (PAS) with and without prior diastase digestion, Mucicarmine and Alcian Blue stains were performed on representative histological sections.

Microscopic examination revealed a relatively homogenous proliferation of average-sized polygonal cells with sharply-defined borders, clear or finely granular cytoplasm and round to oval nuclei with clumped chromatin and prominent nucleoli. Cytoplasmic clearing was consistent across the entire tumor. Nuclear atypia was mild to moderate and the highest mitotic index was 6 mitoses per $10 \mathrm{HPF}$. The neoplastic cells were arranged in compact nodular structures featuring thin and inconspicuous fibro-vascular septa, with focally dilated blood spaces (Fig. 1F). The tumor nests lacked peripheral myoepithelial lining and were closely packed together, forming a well-circumscribed but unencapsulated multinodular structure (Fig. 2A). Focal stromal invasion was noted in the form of solid nests (Fig. 2B). No lympho-vascular invasion or necrosis was noted. There were no proliferative epithelial changes in the surrounding breast tissue, except for non-proliferative fibrocystic changes.

Upon histochemical examination, the vast majority of cells revealed variable granular cytoplasmic positivity in PAS staining, which vanished upon diastase pre-treatment (Fig. 2C). Both Mucicarmine and Alcian Blue stains were negative.

Immunostaining for p63 and smooth muscle myosin revealed limited areas of intraductal component with clear cell features and solid papillary pattern at the periphery of the tumor (Fig. 2D and E). The bulk of the tumor lacked myoepithelial cells both within and at the periphery of the nodules. Tumor cells were strongly and diffusely positive for ER, but completely negative for PR. HER 2 was negative, revealing incomplete membranous staining in $25 \%$ of the tumor cells (score 1+) and Ki67 proliferation index was 5\%. GATA binding protein 3 (GATA3) was also diffusely positive, in compliance with a breast primary tumor. E-cadherin revealed crisp membranous positivity both within the intraductal and invasive components (Fig. 2F). Synaptophysin and chromogranin did not show any neuroendocrine differentiation.

The sentinel lymph node examined in 35 different sections, including routine $\mathrm{H} \& \mathrm{E}$ staining as well as AE1/AE3 and CK7 immunolabeling, revealed reactive follicular hyperplasia, sinus histiocytosis and lipomatous atrophy, with no tumor cells whatsoever.

Based on these findings, the morphological features and immunohistochemical phenotype supported the diagnosis of glycogen-rich clear cell carcinoma of the breast with solid papillary pattern, pT1c pN0 (sn).

A thorough radiological and clinical assessment did not reveal any metastatic foci or other primary tumors. To date, the patient has been followed for 6 months with no evidence of recurrence or metastasis. 


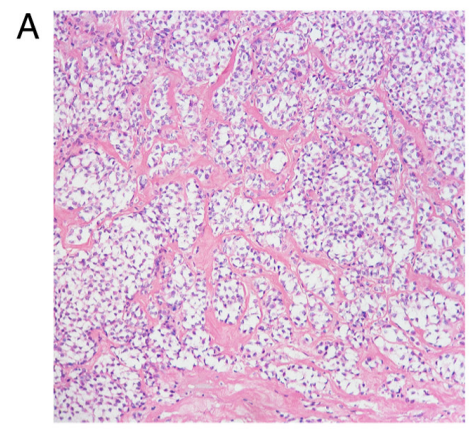

B
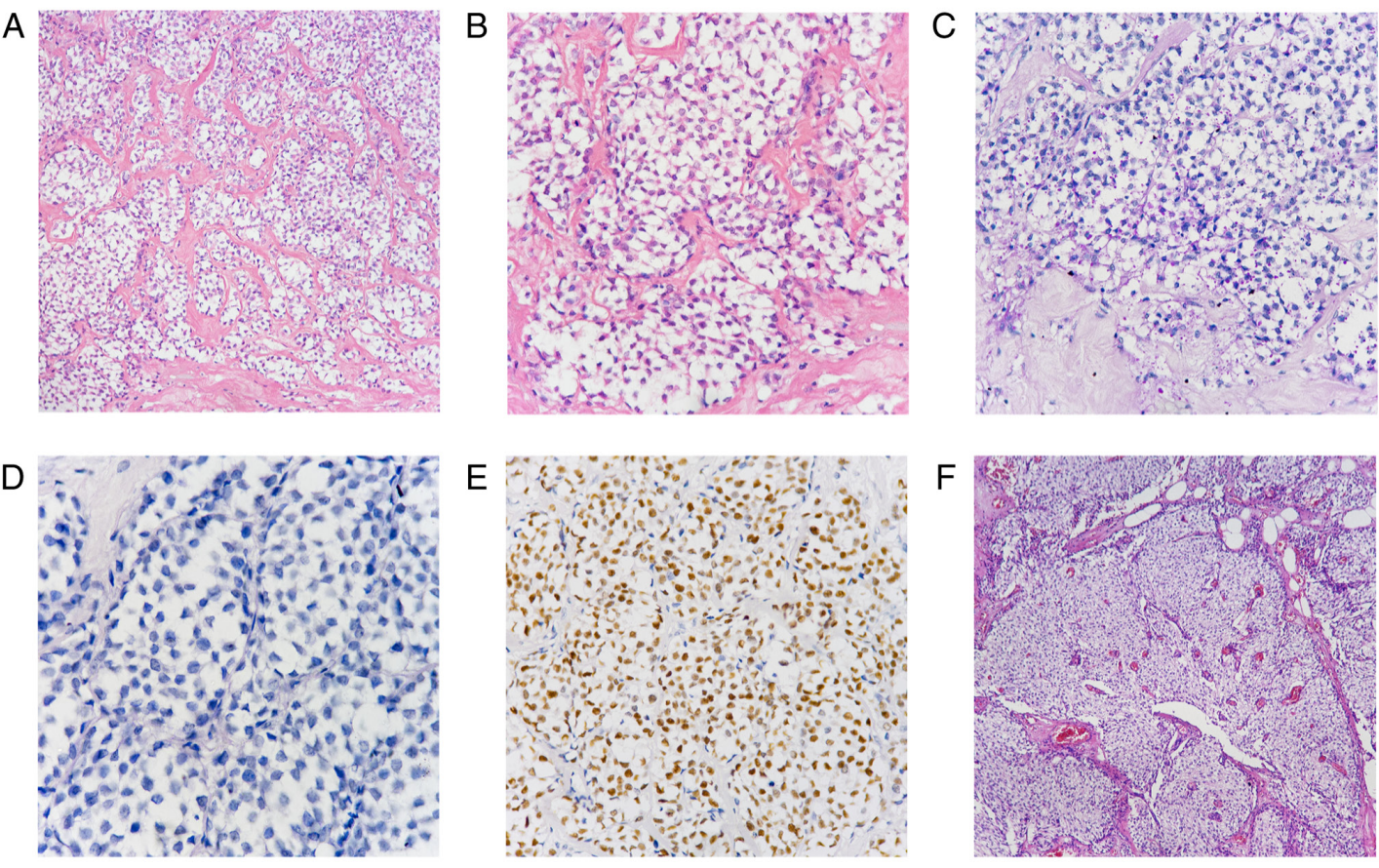

Figure 1. (A) Infiltrative nests, cords or trabecular structures composed of polygonal epithelial cells with well-defined borders and strikingly clear or finely-granular cytoplasm (H\&E stain, magnification, x200). (B) Tumor cell nuclei had irregular shapes and sizes, featuring granular chromatin pattern and prominent mitotic figures (H\&E stain, magnification, $\mathrm{x} 400$ ). (C) Tumor cells showed diffuse finely-granular cytoplasmic positivity in periodic acid-Schiff staining (PAS stain, magnification $\mathrm{x} 400$ ). (D) Finely-granular cytoplasmic positivity of the tumor cells was completely absent in periodic acid-Schiff staining with diastase digestion (PAS-D stain, magnification, x400). (E) Diffuse nuclear positivity for ER in 91-100\% of tumor cells (IHC staining with DAB chromogen, magnification, $\mathrm{x} 400$ ). (F) Bulk of the tumor was composed of nodular structures with thin fibrovascular septa and dilated blood spaces (H\&E stain, magnification, x100). H\&E, hematoxylin and eosin; ER, estrogen receptor; IHC, immunohistochemistry.

\section{Discussion}

Invasive breast carcinoma comprises a heterogenous group of diseases with various pathologic features, genomic alterations, response to treatment and clinical outcome. Most tumors are thought to derive from the terminal ductal lobular unit and up to $75 \%$ of invasive breast carcinomas are classified as ductal carcinomas. The remaining $25 \%$ of breast carcinomas are divided into special histologic subtypes, based on the presence of various characteristic morphologic features (2).

Glycogen-rich clear cell carcinoma (GRCCC) is an extremely rare subtype of breast carcinoma, accounting for anywhere between 0.1 and $3 \%$ of all invasive breast cancers (7). The actual incidence varies from study to study, but most authors agree that it represents a very uncommon morphologic pattern of invasive breast carcinoma of no special type.

According to the World Health Organization definition, GRCCC is a morphological subtype of breast carcinoma in which more than $90 \%$ of the tumor cells feature clear or finely-granular cytoplasm with diastase-sensitive glycogen deposits (2). Tumor cells have moderate to marked nuclear atypia, well-defined cell borders and may be arranged in sheets, nests, cords, solid, papillary, micropapillary, cribriform, alveolar or tubular structures. Some authors also report neuroendocrine, apocrine and mucinous differentiation (4,8-10). Most cases of GRCCC are invasive tumors. Pure intraductal GRCCCs are exceptionally rare $(11,12)$.

GRCCC usually affects women in the 5th decade of life (13). Patients may present with tumor mass, skin dimpling, nipple retraction or pain. Tumor diameter usually ranges between 1 and $6.5 \mathrm{~cm}$ (14), but can reach up to $15 \mathrm{~cm}$ (14) or more. Eun et al recently published a review of radiologic features of GRCCC (15). Based on their findings, GRCCC usually presents as an oval-shaped spiculated tumor mass with irregular margins and fine calcifications (8,16-19). However, mammography may sometimes be inconclusive due to dense breast tissue (20) or may feature benign characteristics (21).

The first case of GRCCC was described by Hull et al in 1981 (3). To the best of our knowledge, only three glycogen-rich breast carcinomas with solid papillary pattern have been reported to date $(3,4,22)$. Case 2 encountered in our practice was extremely similar to the case reported by Hull et al featuring separate areas of solid papillary growth and invasive breast carcinoma (3).

An article published by Gürbüz and Özkara in 2003 was the first publication to detail the immunophenotypical profile of GRCCC, with focus on the differential diagnosis with clear cell renal cell carcinoma (CCRCC). Both tumors may show positivity for CK7, CK8/18 and CK19. Coexpression of vimentin and cytokeratin is a classic feature in CCRCC, but it can also be identified in high grade invasive ductal carcinomas. The absence of ER and PR expression may be interpreted as a feature of non-breast carcinomas, but can also be seen in high grade breast carcinomas. HMWCK 34 $312 \mathrm{E}$ (pan-epithelial cytokeratin including CK1, CK5, CK10 and CK14) is expressed by all epithelial cells of the breast and has been reported to be rarely positive in CCRCC (22). Currently, we benefit from several immunohistochemical markers which can accurately differentiate between a GRCCC primary to the breast and a CCRCC. Mammaglobin, gross cystic disease fluid protein 15 

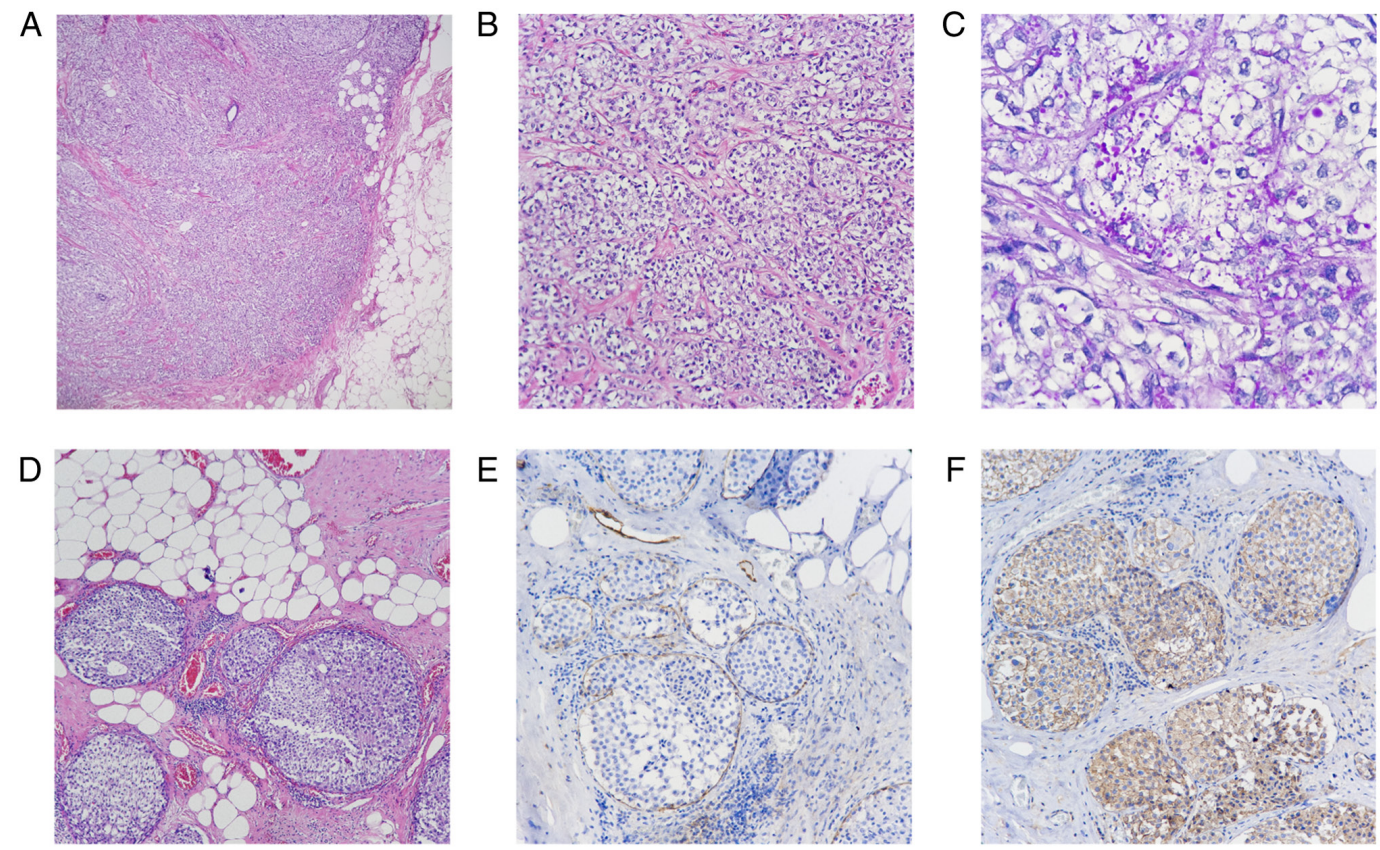

Figure 2. (A) The periphery of the tumor mass well-defined, with pushing borders infiltrating the surrounding fat (H\&E stain, magnification, $\mathrm{x} 40$ ). (B) Stromal invasion was noted in the form of solid nests, similar to the ones observed in the first case (H\&E stain, magnification, $\mathrm{x} 100)$. (C) Tumor cells showed diffuse finely-granular cytoplasmic positivity in periodic acid-Schiff staining (PAS stain, magnification, x400). (D) Periphery of the tumor showed areas of in situ glycogen-rich clear cell carcinoma (H\&E stain, magnification, x200). (E) The in situ component revealed a continuous layer of myoepithelial cells at the periphery, demonstrated by smooth muscle myosin (SMM) staining (IHC with DAB chromogen, magnification, x200). (F) Both the in situ and invasive areas revealed crisp membranous positivity for E-cadherin (IHC with DAB chromogen, magnification, x200). H\&E, hematoxylin and eosin; IHC, immunohistochemistry.

(GCDFP-15) and GATA3 are positive in GRCCC and negative in CCRCC. On the other hand, renal cell carcinoma (RCC), paired-box gene 8 (PAX8) and cluster of differentiation 10 (CD10) are positive in CCRCC and negative in GRCCC. However, it is important to emphasize that from a morphological standpoint, GRCCC of the breast retains the fundamental characteristics of clear cell tumors from other organs.

Normal breast histology may feature clear cell areas as a consequence of physiological changes occurring during pregnancy (14). In regards to breast tumors with clear cell features, the differential diagnosis of GRCCC includes secretory carcinoma, lipid-rich carcinoma, apocrine carcinoma and mucinous carcinoma (23). Cutaneous tumors with clear cell morphology such as clear cell hidradenoma, sebaceous neoplasms and malignant melanoma may involve the breast primarily or secondarily and may be considered in the differential diagnosis. On the other hand, glycogen rich clear cell tumors can also arise and metastasize from other organs such as lung, endometrium, salivary gland, ovary and uterine cervix $(13,20,22,24,25)$. In most cases, an appropriate immunohistochemical panel can identify the cell lineage and special stains such as PAS and PAS-D can confirm the presence of intracytoplasmic glycogen. Molecular techniques are not required in order to establish this diagnosis, but may also play an important role, based on the morphological features of the tumor. For example, detection of $\mathrm{t}(12 ; 15)(\mathrm{p} 13 ; \mathrm{q} 25)$ chromosomal translocation results in the ETV6-NTRK3 fusion gene, which is specific for secretory carcinoma.

Differential diagnosis with other mammary tumors with clear cells is also challenging. For example, clear cell 'sugar' tumor, a tumor with perivascular epithelioid differentiation (PEComa), is characterized by a solid proliferation of epithelioid tumor cells with glycogen-rich clear cytoplasm. This neoplasm has originally been described in the lung, but has recently been reported in extra-pulmonary sites as well, including the breast. Unlike our two cases of GRCCC, the immunophenotype of PEComa is characterized by intense positivity for HMB45, variable expression of muscle markers and complete absence of cytokeratins (26).

Many authors agree that the immunophenotype of GRCCC tends to be ER-positive, PR-negative and HER2-negative (27). Apart from steroid receptors and HER2, the molecular features of GRCCC are largely unknown. However, multiple authors have reported ER-negative, PR-negative, HER2 positive cases (28) as well as triple-negative cases (27), which suggests that GRCCC is almost as heterogenous as IBC-NST. Kuroda et al published a series of 20 cases in which $56 \%$ were luminal A, $12 \%$ were luminal B and $32 \%$ were triple-negative (13). The largest study to date, based on the Surveillance, Epidemiology and End Results (SEER) database, including a total of 155 cases, identified $45 \%$ grade 3 carcinomas with triple-negative phenotype (7).

Due to the rarity of GRCCC of the breast (less than 160 cases to date, mainly as isolated reports or short series), the overall prognosis of this tumor is still controversial. Conventional wisdom holds that the outcome of patients with GRCCC of the breast is not favorable and is probably at least the same or worse than that of IBC-NST when compared on a stage-matched basis $(13,20)$. The SEER database study including data from 1973 to 2015 concluded that GRCCC is an aggressive tumor with poorer prognosis irrespective of AJCC stage, tumor grade, patient age, treatment and ER/PR/HER2 status (7). Hull and Warfel reported that 5 of 10 patients with GRCCC died of metastatic disease (24). Toikkanen and Joensuu reported that 5 of 6 patients presented with axillary 
lymph node metastases at diagnosis and died of the disease within 7 years (5). Fisher et al reported a poorer outcome among 45 patients with GRCCC, greater frequency of nodal metastasis, and higher histologic grade when compared to 1,510 cases of non-clear cell breast carcinoma (6). On the other hand, Ma et al reported 24 cases with no significant difference in overall survival between GRCCC and control invasive breast carcinomas when matched by age, tumor size, nodal status, and immune-phenotype (29). Hayes et al reported 21 cases of GRCCC and also found that prognosis was not different from non-GRCCC when the tumor was matched by size, grade, and lymph node status (4). Our two cases revealed divergent patient outcome as well. The first case showed a highly infiltrative tumor, with lymph node and distant metastases. The patient died within 16 months of metastatic disease. The second case showed a well-defined breast mass with solid papillary architecture and no sentinel lymph node metastasis. The patient is well today and has had no clinical recurrence of the disease after 6 months of follow-up without radiotherapy or chemotherapy. The prognosis is probably better if the tumor is detected early and if there are no lymph node metastases.

In conclusion, GRCCC is an extremely rare breast cancer subtype with controversial prognosis. While some authors report no significant difference in survival when stage-matched with IBC-NST, others observed poorer outcome in patients with GRCCC. After correlating contradictory literature data with our clinical practice findings, we conclude that GRCCC is a heterogenous subtype of breast cancer with a wide morphological spectrum and variable clinical outcome which should be managed similarly to IBC-NST. While some morphological features such as solid papillary pattern may hint at a more favorable outcome, similar to IBC-NST, these are simply speculations which warrant further study. We believe that to date, the molecular phenotype remains the main variable with clinical outcome significance and further molecular studies would not only enhance the knowledge on GRCCC but would also pave the way for novel treatment modalities for this peculiar mammary malignancy.

\section{Acknowledgements}

Not applicable.

\section{Funding}

No funding was received.

\section{Availability of data and materials}

Further information regarding the case reports is available from the corresponding author on reasonable request.

\section{Authors' contribution}

TAG, OM and ACL had substantial contributions to the conception of the article and the acquisition of clinical data. TT, DC, OT, NS and REB had substantial contributions to the analysis and interpretation of data. All the authors were involved in writing the manuscript and revised the manuscript critically for important intellectual content, approved the final version to be published and agreed to be accountable for all aspects of the work in ensuring that questions related to the accuracy or integrity of any part of the work are resolved.

\section{Ethics approval and consent to participate}

The present report was conducted in accordance with the Declaration of Helsinki, and written informed consent was obtained from all patients prior to enrollment in the analysis.

\section{Patient consent for publication}

Patient consent for the publication of the images was obtained before publication.

\section{Competing interests}

The authors declare that they have no competing interests.

\section{References}

1. Thondavadi SR, Krishnamurthy J and Gubbanna VM: A case report of glycogen-rich clear cell carcinoma of breast. Indian J Pathol Microbiol 53: 374-375, 2010.

2. Allison KH, Brogi E and Ellis IO: WHO Classification of Tumours: Breast Tumours: 5th edition. International Agency for Research on Cancer, Lyon, pp102-109, 2019.

3. Hull MT, Priest JB, Broadie TA, Ransburg RC and McCarthy LJ: Glycogen-rich clear cell carcinoma of the breast: A light and electron microscopic study. Cancer 48: 2003-2009, 1981.

4. Hayes MM, Seidman JD and Ashton MA: Glycogen-rich clear cell carcinoma of the breast: A clinicopathologic study of 21 cases. Am J Surg Pathol 19: 904-911, 1995.

5. Toikkanen S and Joensuu H: Glycogen-rich clear-cell carcinoma of the breast: A clinicopathologic and flow cytometric study. Hum Pathol 22: 81-83, 1991.

6. Fisher ER, Tavares J, Bulatao IS, Sass R and Fisher B: Glycogen-rich, clear cell breast cancer: With comments concering other clear cell variants. Hum Pathol 16: 1085-1090, 1985.

7. Zhou Z, Kinslow CJ, Hibshoosh H, Guo H, Cheng SK, He C, Gentry MS and Sun RC: Clinical features, survival and prognostic factors of glycogen-rich clear cell carcinoma (GRCC) of the breast in the U.S. Population. J Clin Med 8: 246, 2019.

8. Sørensen FB and Paulsen SM: Glycogen-rich clear cell carcinoma of the breast: A solid variant with mucus. A light microscopic, immunohistochemical and ultrastructural study of a case. Histopathology 11: 857-869, 1987.

9. Di Tommaso L, Pasquinelli G, Portincasa G and Santini D: Glycogen-rich clear-cell breast carcinoma with neuroendocrine differentiation features. Pathologica 93: 676-680, 2001 (In Italian).

10. Ionescu CA, Matei A, Navolan D, Dimitriu M, Bohâltea R, Neacsu A, Ilinca C and Ples L: Correlation of ultrasound features and the Risk of Ovarian Malignancy Algorithm score for different histopathological subtypes of benign adnexal masses. Medicine (Baltimore) 97: e11762, 2018.

11. Salemis NS: Intraductal glycogen-rich clear cell carcinoma of the breast: A rare presentation and review of the literature. Breast Care (Basel) 7: 319-321, 2012.

12. Seki H, Sasaki K, Morinaga S, Asanuma F, Yanaihara H, Kaneda M, Suzuki K, Ishii Y, Kamiya N, Osaku M and Ikeda T: A case of glycogen-rich clear cell carcinoma of the breast with extensive intraductal components and micrometastases to the axillary lymph node. Gan To Kagaku Ryoho 43: 239-241, 2016 (In Japanese).

13. Kuroda H, Sakamoto G, Ohnisi K and Itoyama S: Clinical and pathological features of glycogen-rich clear cell carcinoma of the breast. Breast Cancer 12: 189-195, 2005.

14. Ratti V and Pagani O: Clear cell carcinoma of the breast: A rare breast cancer subtype-case report and literature review. Case Rep Oncol 8: 472-477, 2015. 
15. Eun NL, Cha YJ, Son EJ, Gweon HM, Kim JA and Youk JH: Clinical imaging of glycogen-rich clear cell carcinoma of the breast: A case series with literature review. Magn Reson Med Sci 18: 238-242, 2019.

16. Takekawa Y, Kubo A, Morita T, Kameda K, Kimura M, Sakakibara M, Yoshii R and Yamashita Y: Histopathological and immunohistochemical findings in a case of glycogen-rich clear cell carcinoma of the breast. Rinsho Byori 54: 27-30, 2006.

17. Pak I, Kutun S, Celik A, Alyanak A, Ardic F and Cetin A: Glycogen-rich 'clear cell' carcinoma of the breast. Breast J 11: $288,2005$.

18. Trupiano JK, Ogrodowczyk E and Bergman S: Pathologic quiz case: Mass in the right breast. Glycogen-rich clear cell carcinoma of the breast. Arch Pathol Lab Med 127: 1629-1630, 2003.

19. Satoh F, Umemura S, Itoh H, Miyajima Y, Tokuda Y, Tajima T and Osamura RY: Fine needle aspiration cytology of glycogen-rich clear cell carcinoma of the breast: A case report. Acta Cytol 42: 413-418, 1998.

20. Mizukami Y, Takayama T, Takemura A, Ichikawa K, Onoguchi M and Taniya T: Glycogen-rich clear cell carcinoma of the breast: A case report. J Med Ultrason 36: 39-43, 2009.

21. Markopoulos C, Mantas D, Philipidis T, Kouskos E, Antonopoulou Z, Hatzinikolaou ML and Gogas H: Glycogen-rich clear cell carcinoma of the breast. World J Surg Oncol 6: 44, 2008.

22. Gürbüz Y and Ozkara SK: Clear cell carcinoma of the breast with solid papillary pattern: A case report with immunohistochemical profile. J Clin Pathol 56: 552-554, 2003.
23. Yerushalmi R, Hayes MM and Gelmon KA: Breast carcinoma-Rare types: Review of the literature. Ann Oncol 20: 1763-1770, 2009.

24. Hull MT and Warfel KA: Glycogen-rich clear cell carcinomas of the breast. A clinicopathologic and ultrastructural study. Am J Surg Pathol 10: 553-559, 1986.

25. Badea M, Baroş A, Bohîlţea RE, Julea IE, Furtunescu FL, Istrate-Ofițeru AM, Iovan L, Cîrstoiu MM, Burcin MR Turcan N, et al: Modern interdisciplinary monitoring of cervical cancer risk. Rom J Morphol Embryol 60: 469-478, 2019.

26. Govender D, Sabaratnam RM and Essa AS: Clear cell 'sugar' tumor of the breast: Another extrapulmonary site and review of the literature. Am J Surg Pathol 26: 670-675, 2002.

27. Vranic S, Skenderi F, Beslagic V and Gatalica Z: Glycogen-rich clear cell carcinoma of the breast: A comprehensive review. Appl Immunohistochem Mol Morphol 28: 655-660, 2020.

28. Sinha KP, Bharati P. Glycogen rich clear cell carcinoma of the breast: a rare case report. IOSR J Dental Medical Sci 15: 85-87, 2016. e-ISSN: 2279-0853, p-ISSN: 2279-0861. Ver. VIII.

29. Ma X, Han Y, Fan Y, Cao X and Wang X: Clinicopathologic characteristics and prognosis of glycogen-rich clear cell carcinoma of the breast. Breast J 20: 166-173, 2014.

This work is licensed under a Creative Commons

Attribution-NonCommercial-NoDerivatives 4.0 International (CC BY-NC-ND 4.0) License. 